\title{
Progressive Multifocal Leukoencephalopathy after Treatment with Nivolumab
}

\section{Martin Martinot, Guido Ahle, Inesa Petrosyan, Camille Martinez, Dragos M. Gorun, Mahsa Mohseni-Zadeh, Samira Fafi-Kremer, Martine Tebacher-Alt}

Author affiliations: Hôpitaux Civils de Colmar, Colmar, France (M. Martinot, G. Ahle, I. Petrosyan, C. Martinez, D.M. Gorun, M. Mohseni-Zadeh); Hôpitaux Universitaires de Strasbourg, Strasbourg, France (S. Fafi-Kremer, M. Tebacher-Alt)

DOI: https://doi.org/10.3201/eid2408.180460

Progressive multifocal leukoencephalopathy (PML) is increasingly being reported in patients undergoing immunotherapy. We report a case of progressive multifocal leukoencephalopathy after treatment with nivolumab, a PD-1 blocker that is used to restore impaired T-cell responses in patients with cancer and infections. Data for 4 other cases were obtained from pharmacovigilance databases.

$\mathrm{P}^{\mathrm{i}}$ rogressive multifocal leukoencephalopathy (PML) is a life-threatening disease of the central nervous system caused by JC polyoma virus (JCV). Although incidence of this disease reportedly has increased markedly in association with HIV/AIDS, a new complication caused by immune-mediated therapies, particularly monoclonal antibodies, has emerged (1).

Nivolumab, a cancer immunotherapy, is a checkpoint inhibitor that functions by blocking the programmed cell death 1 (PD-1)/programmed death ligand 1 pathway and restoring immunity against tumor cells (2). We report a case of PML that occurred after 12 months of treatment with nivolumab in a patient with refractory stage IV Hodgkin lymphoma.

A 54-year-old HIV-negative man was hospitalized in November 2017 for new-onset acute back pain that was not responsive to high-dose morphine. In 2013, he had been given a diagnosis of stage IV Hodgkin lymphoma, for which he underwent several chemotherapy sessions, including ABVD (adriamycin, bleomycin, vinblastine, dacarbazine); DHAP (dexamethasone, high-dose cytarabin, cisplatin); ICE (ifosfamide, carboplatin, etoposide); ChIVPP (chlorambucil, vinblastine, procarbazine, prednisolone); etoposide, gemcitabine, liposomal doxorubicin plus methylprednisolone; and brentuximab plus bendamustin. In 2016, a positron emission tomography scan showed new locations of lymphoma (lymph nodes, spine, and lungs).

He was then given nivolumab ( $3 \mathrm{mg} / \mathrm{kg}$ every $2 \mathrm{wk}$ ) during October 2016-November 2017. He was also taking hydrocortisone $(30 \mathrm{mg} / \mathrm{d})$ for hypocorticism; $110 \mathrm{mg} / \mathrm{d}$ starting in March 2017). At admission, corticosteroid therapy was increased to $40 \mathrm{mg} / \mathrm{d}$ of methylprednisolone to treat hyperalgesic spine locations.

Three weeks later, progressive hemiparesis developed on the left side of his body. Magnetic resonance imaging of the brain showed multiple nonenhancing lesions that were suggestive of PML (Figure). Analysis of cerebrospinal fluid showed identical concentrations of leukocytes and erythrocytes $\left(1 \mathrm{cell} / \mathrm{mm}^{3}\right)$. PCR analyses of showed a JCV concentration of 2,230 copies/mL in cerebrospinal fluid and 9,720 copies/mL in blood, confirming the diagnosis of PML.

Nivolumab was discontinued, and corticosteroid dose was decreased. CD4 lymphocyte subpopulation counts were 227 cells $/ \mathrm{mm}^{3}$ (12\%) and CD8 lymphocyte subpopulation counts 1,547 cells $/ \mathrm{mm}^{3}(83 \%)$. The patient was still alive 5 months later but showed no neurologic improvement and had persistent JCV viremia (2,506 copies/mL).

PD-1 is a transmembrane receptor expressed on dendritic, NK, CD4, CD8 and T-cells and involved in downregulation of the immune system by promotion of activated T-cell apoptosis and diminution of regulatory T-cell apoptosis to stop unrestrained cytotoxic functions $(3,4)$. Blockade of pathways involving PD-1 and its ligand is a promising treatment for cancers (melanoma, nonsmall cell lung cancer, metastatic renal cell carcinoma, head and neck carcinomas, Hodgkin lymphoma, and urothelial carcinoma) (4), although the role of PD1/programmed death ligand 1 in immune suppression and the mechanism of action of antibodies remain to be better defined.

Checkpoint inhibitors can cause unique toxicities, known as immune-related adverse events (IRAEs), including rashes, colitis, hepatitis, nephritis, pneumonitis, and thyroid disturbance, but these rarely comprise neurologic manifestations $(<1 \%)$ such as aseptic meningitis and immune encephalitis $(3,5,6)$. Neurologic IRAEs occur rapidly after treatment with checkpoint inhibitors and disappear after treatment interruption and introduction of steroids (6). Therefore, we excluded a diagnosis of neurologic IRAEs for this patient. Infections related to treatment with checkpoint inhibitors are infrequent (4).

Checkpoint inhibitors have been studied as treatment for chronic infectious diseases by restoring T-cell depletion $(2,3,7)$, including PML, in which PD-1 on CD4 and CD8 cells has been reported $(1,8)$. In a series of 740 patients given checkpoint inhibitors, serious infections developed in $7.4 \%$, particularly in patients taking ipilimumab and nivolumab, steroids, or tumor necrosis factor antibodies for treatment of IRAEs, but few opportunistic infections and no PML were reported (9).

We found 4 unpublished cases of PML related to nivolumab in pharmacovigilance databases, but few data are available for these cases. One case of PML in the 


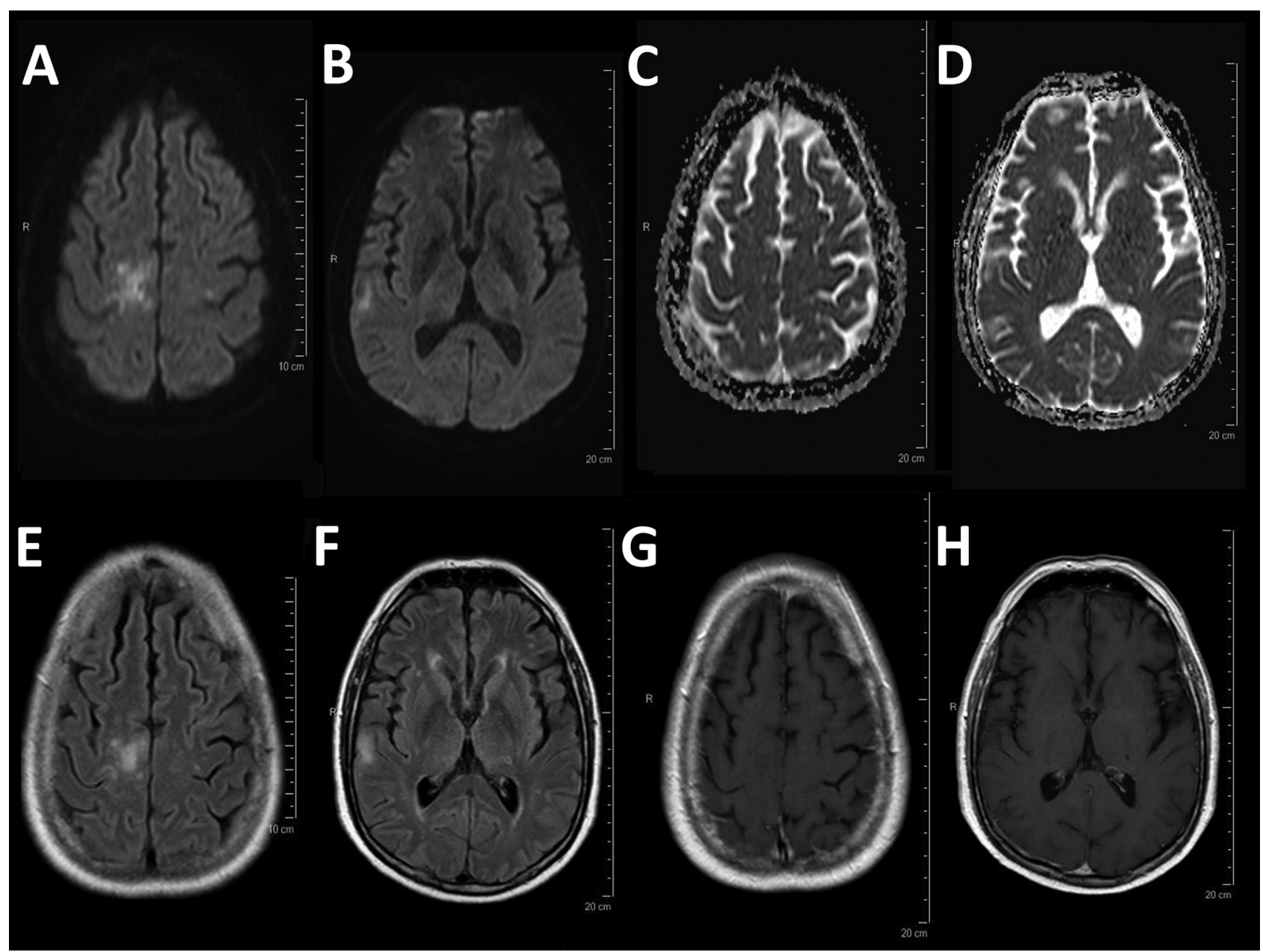

Figure. Magnetic resonance imaging for a 54-year-old man with progressive multifocal leukoencephalopathy after treatment with nivolumab, showing typical multifocal lesions: diffusion weighted imaging hyperintensity $(A, B)$ with no decrease in the apparent diffusion coefficient $(C, D)$, corresponding patchy corticosubcortical hyperintensities on fluid-attenuated inversion recovery image (E, $F$ ) without enhancement on T1-weighted imaging after administration of gadolinium $(G, H)$.

European database EudraVigilance did not contain any detailed information. We found 3 cases in the World Health Organization pharmacovigilance database: a 70-yearold man with metastatic melanoma who was treated with ipilimumab for 3 months and nivolumab for 9 months; a 61-year-old woman with lung cancer who was given nivolumab (300 mg) 21 days before PML developed; and a 61 -year-old woman who was treated with nivolumab (300 mg every $15 \mathrm{~d}$ for $5 \mathrm{mo}$ ).

Another reported mechanism of development of infections with use of checkpoint inhibitors is restoration of T-cell function that mimics immune restoration syndrome (IRIS), which can cause reactivation of latent tuberculosis (4) and acute progression of aspergillosis. IRIS has been reported in patients with PML, but for our patient, use of steroids and onset of PML long after treatment with nivolumab was started did not support a diagnosis of IRIS.
This patient was highly immunocompromised because of lymphoma and use of steroids. Thus, it is difficult to determine whether nivolumab contributed to development of PML or whether PML was caused by combinations of different factors. PML cases have been reported even long after cure of the initial condition, but the incidence of PML among patients with hematologic malignancies is rare $(0.07 \%)(10)$. However, because of the complexity and unknown mechanisms of checkpoint inhibitors and reported cases of PML, physicians should be vigilant when using this treatment.

\section{About the Author}

Dr. Martinot is head of the Infectious Diseases Unit, Colmar Hospital, Colmar, France. His primary research interests are tickborne diseases, influenza, and infections in immunocompromised patients. 


\section{References}

1. Misbah SA. Progressive multi-focal leukoencephalopathy: driven from rarity to clinical mainstream by iatrogenic immunodeficiency. Clin Exp Immunol. 2017;188:342-52. http://dx.doi.org/10.1111/ cei.12948

2. Dyck L, Mills KHG. Immune checkpoints and their inhibition in cancer and infectious diseases. Eur J Immunol. 2017;47:765-79. http://dx.doi.org/10.1002/eji.201646875

3. McCarthy MW, Walsh TJ. Checkpoint inhibitors and the risk of infection. Expert Review of Precision Medicine and Drug Development. 2017;2:287-93. http://dx.doi.org/10.1080/ 23808993.2017.1380517

4. Redelman-Sidi G, Michielin O, Cervera C, Ribi C, Aguado JM, Fernández-Ruiz M, et al. ESCMID Study Group for Infections in Compromised Hosts (ESGICH) Consensus Document on the safety of targeted and biological therapies: an infectious diseases perspective (immune checkpoint inhibitors, cell adhesion inhibitors, sphingosine-1-phosphate receptor modulators and proteasome inhibitors). Clin Microbiol Infect. 2018; S1198-743X(18)30148-4.

5. Costa R, Costa RB, Talamantes SM, Helenoswki I, Carneiro BA, Chae YK, et al. Analyses of selected safety endpoints in phase 1 and late-phase clinical trials of anti-PD-1 and PD-L1 inhibitors: prediction of immune-related toxicities. Oncotarget. 2017;8:677829. http://dx.doi.org/10.18632/oncotarget.18847

6. Cuzzubbo S, Javeri F, Tissier M, Roumi A, Barlog C, Doridam J, et al. Neurological adverse events associated with immune checkpoint inhibitors: review of the literature. Eur J Cancer. 2017;73:1-8. http://dx.doi.org/10.1016/j.ejca.2016.12.001

7. Rao M, Valentini D, Dodoo E, Zumla A, Maeurer M. Anti-PD-1/ PD-L1 therapy for infectious diseases: learning from the cancer paradigm. Int J Infect Dis. 2017;56:221-8. http://dx.doi.org/ 10.1016/j.ijid.2017.01.028

8. Tan CS, Bord E, Broge TA Jr, Glotzbecker B, Mills H, Gheuens $\mathrm{S}$, et al. Increased program cell death-1 expression on $\mathrm{T}$ lymphocytes of patients with progressive multifocal leukoencephalopathy. J Acquir Immune Defic Syndr. 2012;60:244 8. http://dx.doi.org/10.1097/QAI.0b013e31825a313c

9. Del Castillo M, Romero FA, Argüello E, Kyi C, Postow MA, Redelman-Sidi G. The spectrum of serious infections among patients receiving immune checkpoint blockade for the treatment of melanoma. Clin Infect Dis. 2016;63:1490-3. http://dx.doi.org/ 10.1093/cid/ciw539

10. Carson KR, Evens AM, Richey EA, Habermann TM, Focosi D, Seymour JF, et al. Progressive multifocal leukoencephalopathy after rituximab therapy in HIV-negative patients: a report of 57 cases from the Research on Adverse Drug Events and Reports project. Blood. 2009;113:4834-40. http://dx.doi.org/10.1182/ blood-2008-10-186999

Address for correspondence: Martin Martinot, Medecine Interne et Rhumatologie, Hôpital Pasteur, 39 Ave de la Liberte, Colmar 68024, France; email: martin.martinot@ch-colmar.fr

\section{Isolation of Candida auris from Ear of Otherwise Healthy Patient, Austria, 2018}

\author{
Shiva Pekard-Amenitsch, Agnes Schriebl, \\ Wilhelm Posawetz, Birgit Willinger, Bettina Kölli, \\ Walter Buzina
}

Author affiliations: AGES-Austrian Agency for Health and Food Safety, Graz, Austria (S. Pekard-Amenitsch, A. Schriebl); Private otorhinolaryngology practice, Graz (W. Posawetz); Medical University of Vienna, Vienna, Austria (B. Willinger); Medical University Graz, Graz (B. Kölli, W. Buzina)

DOI: https://doi.org/10.3201/eid2408.180495

The emerging pathogen Candida auris is isolated mostly from hospitalized patients and often shows multidrug resistance. We report on the isolation of this yeast in Austria from an outpatient's auditory canal. The isolate showed good susceptibility against antifungals except for echinocandins; the patient was treated successfully with topical administration of nystatin.

$\mathrm{T}$ he yeast Candida auris was first isolated from the external auditory canal of a person in Japan in 2009 (1). Reports from all continents except Australia (2) exist, but an outbreak in Europe was reported only recently, from a hospital in London (3). Major outbreaks of C. auris from countries in Europe have been reported from the United Kingdom and Spain, and sporadic isolations have been reported in Germany, France, Belgium, and Norway $(2,4)$. We report on an isolation of $C$. auris in Austria, cultivated from an infection of the external auditory canal (otitis externa).

In January 2018, an otherwise healthy 22-year-old man came to an established otorhinolaryngology practice with therapy-refractory otitis externa in both ears that had persisted for almost 4 years despite antimicrobial drug treatment. The patient is an Austrian citizen with Turkish ancestry. He used to travel to Turkey frequently; his last visit was in 2017, and other trips abroad were not reported. A smear test was taken and sent to a microbiological laboratory for bacterial examination. A yeast grew after 48 hours at $37^{\circ} \mathrm{C}$; the yeast was transferred to chromogenic mycological media, and an intense pink-colored yeast was cultivated on Brilliance Candida Agar (Oxoid, Basingstoke, UK). The isolate showed a pale rose color on BBL CHROMagar Candida Medium (Becton Dickinson, Heidelberg, Germany), and on Candida ID medium (bioMérieux, Marcy l'Étoile, France) (online Technical Appendix Figure, https://wwwnc.cdc.gov/EID/ article/24/8/18-0495-Techapp1.pdf). On Sabouraud glucose agar and on malt extract agar (both from Becton Dickinson), the colonies were white. The maximum temperature at 